\title{
BMJ Open Consensus-based approach to managing opioids, including opioid misuse and opioid use disorder, in patients with serious illness: protocol for a modified Delphi process
}

\author{
Caroline King (D) , ${ }^{1}$ Robert Arnold, ${ }^{2}$ Emily Dao, ${ }^{3}$ Jennifer Kapo, ${ }^{4}$ Jane Liebschutz, ${ }^{5}$ \\ Diane Meier, ${ }^{6}$ Judith Paice, ${ }^{7}$ Christine Ritchie, ${ }^{8}$ Kristen Czajkowski, ${ }^{2,5}$ \\ Dmitry Khodyakov, ${ }^{3}$ Jessica Merlin ${ }^{2,5}$
}

To cite: King C, Arnold R, Dao E, et al. Consensusbased approach to managing opioids, including opioid misuse and opioid use disorder, in patients with serious illness: protocol for a modified Delphi process. BMJ Open 2021;11:e045402. doi:10.1136/ bmjopen-2020-045402

- Prepublication history for this paper is available online. To view these files, please visit the journal online (http://dx.doi. org/10.1136/bmjopen-2020045402).

Received 30 September 2020 Accepted 04 May 2021

Check for updates

\section{(C) Author(s) (or their} employer(s)) 2021. Re-use permitted under CC BY-NC. No commercial re-use. See rights and permissions. Published by BMJ.

For numbered affiliations see end of article.

Correspondence to

Caroline King; kingca@ohsu.edu

\section{ABSTRACT}

Introduction Management of opioid misuse and opioid use disorder (OUD) among individuals with serious illness is an important yet understudied issue. Palliative care clinicians caring for individuals with serious illness, many of whom may live for months or years, describe a complex tension between weighing the benefits of opioids, which are considered a cornerstone of pain management in serious illness, and serious opioid-related harms like opioid misuse and OUD. And yet, little literature exists to inform the management of opioid misuse and OUDs among individuals with serious illness. Our objective is to provide evidence-based management guidance to clinicians caring for individuals with serious illness who develop opioid misuse or OUD.

Methods and analysis We chose a modified Delphi approach, which is appropriate when empirical evidence is lacking and expert input must be used to shape clinical guidance. We sought to recruit 60 clinicians with expertise in palliative care, addiction or both to participate in this study. We created seven patient cases that capture important management challenges in individuals with serious illness prescribed opioid therapy. We used ExpertLens, an online platform for conducting modified Delphi panels. Participants completed three rounds of data collection. In round 1, they rated and commented on the appropriateness of management choices for cases. In round 2, participants reviewed and discussed their own and other participants' round 1 numerical responses and comments. In round 3 (currently ongoing), participants again reviewed rounds 1 and 2, and are allowed to change their final numerical responses. We used ExpertLens to automatically identify whether there is consensus, or disagreement, among responses in panels. Only round 3 responses will be used to assess final consensus and disagreement.

Ethics and dissemination This project received ethical approval from the University of Pittsburgh's Institutional Review Board (study 19110301) and the RAND Institutional Research Board (study 2020-0142). Guidance from this work will be disseminated through national stakeholder networks to gain buy-in and endorsement. This study
Strengths and limitations of this study

- This study uses a rigorous modified Delphi approach to provide important guidance on the management of opioid misuse and opioid use disorder among individuals with serious illness, especially advanced cancer.

- Participants are drawn from experts in both palliative care and addiction.

- This Delphi study is being conducted asynchronousIy online, which has the benefit of reducing barriers to participation such as travel and scheduling; however, some participants may find engaging in anonymous discussion online challenging.

- The success of the Delphi approach relies on identifying participants with appropriate expertise. Although we have worked systematically to recruit participants with expertise in palliative care and addiction, it is possible that we may have missed important voices, including those of clinicians outside the USA, or those representing non-academic medical communities. It is also possible that some perspectives may be more fully represented than others.

- Results will be disseminated through peer-reviewed manuscripts and conferences, and ultimately, developed into a nationally distributed implementation toolkit.

will also form the basis of an implementation toolkit for clinicians caring for individuals with serious illness who are at risk of opioid misuse or OUD.

\section{INTRODUCTION}

Management of opioid misuse and opioid use disorder (OUD) among individuals with serious illness, particularly in palliative care settings, is an important yet understudied issue. Serious illnesses include health conditions with a high risk of mortality that 
negatively impact a person's daily function, quality of life or excessively strain their caregivers. ${ }^{1}$ Examples include cancer, heart failure, chronic obstructive pulmonary disease and others. Seriously ill patients often seek pain management at palliative care clinics. ${ }^{2}$ Palliative care focuses on relief of the pain, symptoms and stresses of serious illness for patients and their families, regardless of the stage or severity of illness. ${ }^{3}$ Palliative care clinicians caring for individuals with serious illness, many of whom may live for months or years, describe a complex tension between weighing the benefits of opioids, which are considered a cornerstone of pain management in serious illness, ${ }^{4}$ and serious opioid-related harms, such as opioid misuse and OUD. ${ }^{5-8}$ These harms could arise when an individual prescribed opioids for their pain without any history of misuse/OUD develops these complications. Additionally, patients may have OUD that precedes their cancer diagnosis, potentially increasing the risks associated with opioid pain management. ${ }^{9}$

The literature describing opioid misuse and OUD in palliative care settings or among individuals with serious illness in general is limited but underscores the importance of these harms. A systematic review mostly including studies from primary care or pain clinics suggests that among individuals on long-term opioid therapy for chronic pain found that $21 \%-29 \%$ of individuals on longterm opioids developed opioid misuse, and $8 \%-12 \%$ of individuals on long-term opioids developed OUD. ${ }^{10}$ Not surprisingly, palliative care clinicians also report spending a significant amount of time managing these opioidrelated challenges. ${ }^{11}$

Furthermore, little literature exists to inform the management of opioid misuse and OUDs among individuals with serious illness. For example, cancer is the most common serious illness seen in palliative care settings. ${ }^{2}$ Most research exploring opioid misuse and OUDs focuses exclusively on patients with chronic 'non-cancer' pain and specifically excludes patients with cancer. This reflects a prevalent belief that opioid benefits and harms in patients with cancer are so different from patients without cancer that they merit separate consideration. ${ }^{512}$ However, this exclusion has led to an evidence vacuum. One group has described the evidence base about opioid benefits and harms, which includes management of opioid misuse and OUD, as 'one of the scarcest bodies of literature in cancer'. ${ }^{7}$ We propose to solicit expert opinion using a modified Delphi method to determine the appropriateness of different strategies of opioid misuse management and address this evidence gap. Specifically, our objective is to provide evidence-based management guidance to clinicians caring for individuals with serious illness who develop opioid misuse or OUD.

\section{METHODS AND ANALYSIS}

To solicit expert opinion, we used the RAND/UCLA Appropriateness Method (RAM), also known as the modified Delphi method. ${ }^{13}$ This approach may be used when empirical evidence on a topic is not available, necessitating expert input to shape clinical guidance. ${ }^{14}$ This study uses input from clinicians with expertise in palliative care, addiction or both, to rigorously and iteratively explore the existence of areas of consensus in our area of interest: management of opioid misuse and OUD in patients with serious illness, specifically advanced cancer. RAM panels focus on clinical scenarios and questions about the appropriateness of various treatment or management options. Inclusion of an online discussion round is what makes our study an online modified Delphi, which is an accepted approach to generating evidence for clinical questions. ${ }^{15}$ See figure 1 for an overview of our proposed online modified Delphi study, which we describe in detail here.

\section{Case and management strategy development}

Our team previously conducted two studies that provided insight into how to design cases and responses (clinical management strategies) ${ }^{1617}$ In a recent qualitative study, we asked palliative care clinicians to describe challenges

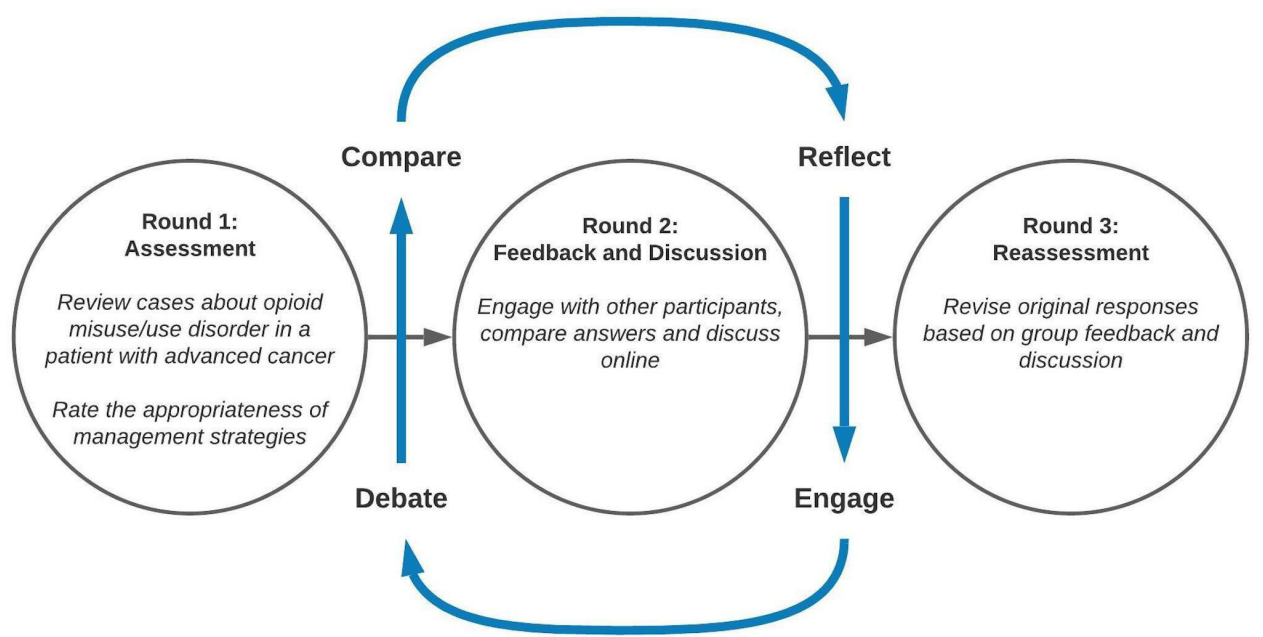

Figure 1 Modified Delphi process for consensus-based approaches to managing opioid-related challenges in patients with serious illness. . $^{29}$ 
they face when caring for patients with serious illness prescribed opioid therapy. Clinical management challenges identified included managing opioids in patients with a history of substance use disorders and addressing opioid-related harms, including misuse behaviours such as concurrent use of cocaine or medications that were not prescribed such as benzodiazepines. ${ }^{16}$ In addition to substance use, other prior work has identified several common and challenging misuse behaviours that may arise in patients prescribed opioid therapy such as taking more opioids than prescribed or aggressive behaviour. ${ }^{17}$ Importantly, our qualitative study consistently identified prognosis for life expectancy (referred to in this article as prognosis) as an important factor that influences opioid decision-making in individuals with serious illness. For example, many participants thought that opioid-related harms may be a less important consideration when prognosis is short. We used these findings as a basis for writing Delphi study cases.

We used these prior studies as a starting point to create cases with challenging clinical characteristics. ${ }^{16}{ }^{17}$ Additionally, given the importance of prognosis in decisionmaking, we decided to present the same cases and management strategies in a hypothetical patient with a prognosis of weeks to months, and separately in a patient with a prognosis of months to years.

All vignettes started with the same basic case with a prognosis of either weeks to months or months to years. The study team decided that this basic case should include attributes that (1) would lead many clinicians to prescribe opioids, (2) are common in ambulatory palliative care and (3) for which opioid therapy would be guidelineconcordant care. $^{18}$ Attributes within final cases described a middle-aged patient of unspecified gender or race, with advanced cancer, undergoing treatment and experiencing pain. This basic case was as follows:

You are seeing a 50-year-old patient with advanced cancer (defined as cancer that is unlikely to be cured or controlled with treatment). They are on active anti-cancer treatment. They have pain related to their cancer or its treatment. The patient's prognosis is weeks-months [in second panel, months-years]. Assume that you have your $\mathrm{X}$ waiver to prescribe buprenorphine/naloxone for opioid use disorder (OUD) and that the patient's insurance covers buprenorphine/naloxone if needed.

Table 1 provides the full text of the cases. The study team prioritised the development of cases that represent particularly common or challenging issues raised in our preliminary study ${ }^{16}$ and in our prior work on opioid misuse behaviours. ${ }^{17}$ Ultimately, we developed seven cases which each added additional clinical information beyond the basic case centering on the following management challenges: (1) inadequate pain control on highest recommended dose of buprenorphine/naloxone, (2) inadequate pain control on stable methadone dose, (3) requests early refills, (4) positive urine drug screen for benzodiazepines that were not prescribed,
(5) positive urine screen for cocaine or methamphetamine, (6) aggressive patient behaviour in clinic and (7) history of untreated OUD not currently on pharmacologic treatment, with unmanaged pain.

Each case was followed by several questions on appropriateness of various management strategies based on published management strategies for opioid misuse behaviours in primary care settings ${ }^{20}$ and study team clinical expertise. These included strategies such as increasing opioids; tapering opioids; starting, splitting and stopping buprenorphine/naloxone, methadone, or other full agonist therapy and referring patients to addiction treatment. Appropriateness was queried using a 9-point Likert scale from the RAM, ${ }^{21}$ which ranges from 'very inappropriate' to 'very appropriate'. Free-text boxes were also provided to allow participants to comment on additional information they would need to inform their management for each case, or provide other relevant thoughts.

The study team piloted these cases and management strategies using a cognitive interviewing-based approach, in which cases and responses were read aloud and assessed for clarity, understanding and content. ${ }^{22}$ Cases and strategies were iteratively refined and then finalised.

\section{Recruitment}

Participants were recruited online from the American Academy of Hospice and Palliative Medicine, Hospice and Palliative Nurses Association, Buprenorphine Clinician Support Network, Society of General Internal Medicine Pain and Addiction Shared Interest Groups, American Academy of Hospice and Palliative Medicine Addiction Shared Interest Group and the Palliative Care Research Cooperative Pain and Opioids Special Interest Group. Although membership in these groups is not limited geographically, these are all US-based groups. A list of additional experts to approach individually was generated by the study team and recommendations from potential participants.

\section{Eligibility criteria}

Potential participants were emailed a survey to determine eligibility. Participants were eligible to participate if they were $>18$ years old and (1) were board-certified in addiction medicine, palliative care or both; (2) had trained (in residency or fellowship) in addiction medicine, palliative care or both or (3) demonstrated other expertise in adult addiction or palliative care (were waivered to prescribe buprenorphine/naloxone for OUD; prescribe buprenorphine/naloxone, methadone or other opioids in palliative care or addiction settings to manage pain or addiction; conduct research related to opioid prescribing in palliative care settings or outpatient OUD treatment or have spoken at national conferences about these topics). Individuals who met eligibility criteria and were willing to participate were prompted to complete a demographics survey that included gender, race, ethnicity, age, expertise, clinical role, time since completion of terminal degree and state of practice. 
Table 1 Cases generated from round 0 for modified Delphi panel exploring opioid therapy in palliative care patients

All cases begin with the following text: 'You are seeing a 50-year-old patient with advanced cancer (defined as cancer that is unlikely to be cured or controlled with treatment). They are on active anti-cancer treatment. They have pain related to their cancer or its treatment. The patient's prognosis is weeks-months [in second panel, months-years]. Assume that you have your X waiver to prescribe buprenorphine/ naloxone for opioid use disorder (OUD) and that the patient's insurance covers buprenorphine/naloxone if needed'.

\section{Case Case scenario}

1 The patient has OUD and is on long-term treatment with daily buprenorphine/naloxone with excellent adherence at the highest dose you would recommend prescribing.

- The patient's pain control is NOT acceptable.

- Assume non-opioid pharmacologic and non-pharmacologic treatments have been maximised and you have provided the patient with appropriate opioid education.

2 The patient has OUD and is on treatment with methadone daily from a methadone clinic at a stable dose with good adherence.

- The patient's pain control is NOT acceptable.

- Assume non-opioid pharmacologic and non-pharmacologic treatments have been maximised and you have provided the patient with appropriate opioid education.

3 The patient does not have a history of an OUD.

- They have been prescribed full agonist opioid(s) (eg, oxycodone, morphine, hydromorphone, fentanyl, methadone dosed three times daily).

- You send appropriate screening and confirmatory urine drug tests, and they are negative for the opioid(s) you prescribed. Other urine drug testing findings are as expected.

- The patient's pain control and function are NOT acceptable.

- The patient reports taking more opioids than prescribed and running out of medications 1 week early, which would explain the negative urine findings. You review the chart and notice this is second time this has happened, and the first time they were educated about the risks of this behaviour and told not to do it again.

- Assume non-opioid pharmacologic and non-pharmacologic treatments have been maximised and you have provided the patient with appropriate opioid education, including asking the patient to call if pain control is inadequate rather than taking more opioids than prescribed.

4 The patient does not have a history of an OUD.

- They have been prescribed full agonist opioid(s) (eg, oxycodone, morphine, hydromorphone, fentanyl and methadone dosed three times daily).

- You send appropriate screening and confirmatory urine drug tests, and they are positive for the opioid(s) you prescribed, and also positive for a benzodiazepine that was not prescribed. You review the chart and notice this is second time this has happened, and the first time they were educated about the risks of this behaviour and told not to do it again.

- The patient reports taking a friend or family member's benzodiazepine for anxiety and sleep.

The patient's pain control and function are acceptable.

Assume you will also fully evaluate and manage the patient's anxiety and sleep concerns, and re-educate the patient about the dangers of taking medications that are not prescribed.

5 The patient does not have a history of an OUD.

- They have been prescribed full agonist opioid(s) (eg, oxycodone, morphine, hydromorphone, fentanyl and methadone dosed three times daily).

- You send appropriate screening and confirmatory urine drug tests, and they are positive for the opioid(s) you prescribed, and also positive for cocaine or methamphetamine. Other urine drug testing findings are as expected.

- The patient's pain control and function are acceptable.

You discuss the urine result with the patient, and they acknowledge recent cocaine or methamphetamine use.

6 The patient does not have a history of an OUD.

They have been prescribed full agonist opioid(s) (eg, oxycodone, morphine, hydromorphone, fentanyl and methadone dosed three times daily).

- The patient exhibits aggressive behaviour where there is a concern for provider or staff safety (eg, threats towards staff). There is no reason to believe there is a medical explanation for the aggressive behaviour.

- The patient's pain control and function are acceptable.

- You educate the patient about appropriate behaviour in the clinic, and they continue to be aggressive.

7 The patient has a recent history of OUD, but they are not currently on medication for OUD (eg, methadone, buprenorphine and naltrexone).

The patient is not currently prescribed any full agonist opioid (eg, oxycodone, morphine, hydromorphone and fentanyl).

- The patient's pain and function are NOT controlled.

- Assume non-opioid pharmacologic and non-pharmacologic treatments have been maximised.

All cases begin with the following text: 'You are seeing a 50-year-old patient with advanced cancer (defined as cancer that is unlikely to be cured or controlled with treatment). They are on active anti-cancer treatment. They have pain related to their cancer or its treatment. The patient's prognosis is weeks-months [in second panel, months-years]. Assume that you have your X waiver to prescribe buprenorphine/naloxone for opioid use disorder (OUD) and that the patient's insurance covers buprenorphine/naloxone if needed'.

OUD, opioid use disorder.

\section{Panel creation}

To minimise participant burden and allow participants to focus on case scenarios that all specify the same prognosis, participants were randomly assigned to participate in either the 'weeks to months' or 'months to years' prognosis panel. During randomisation, we stratified 
by participant expertise in palliative care or addiction. Given fewer numbers of participants with addiction expertise than palliative care expertise, we categorised participants who had both addiction expertise and palliative care expertise as being in the addiction category. We also stratified by professional identity (physician and advanced practice provider such as nurse practitioner). This approach was taken to balance the type of expertise on each panel.

\section{Sample size}

Previous research recommends including 40-60 participants in online Delphi studies. ${ }^{23}$ Our goal was to include a minimum of 40 participants per panel. We aimed to recruit 60 participants per panel to account for attrition.

\section{Data collection}

Data for all three Delphi rounds were collected using ExpertLens, a web-based platform developed by RAND that allows for participation in Delphi panels online. ${ }^{24}$

Round 1 began on 10 August 2020. Participants were asked to rate and comment on the appropriateness of management choices for the seven cases.

Round 2 began on 10 September 2020. The purpose of round 2 was to allow participants to consider other points of view and re-consider their round 1 responses. Participants were asked to review their own and other participants' numerical responses and free-text comments from round 1. For each case and each management strategy, information was provided as to whether consensus was reached, and if consensus was reached whether the strategy was found to be appropriate, not appropriate or of uncertain appropriateness, based on the prespecified analytic approach (see the Data analysis section). Participants also viewed summaries of round 1 free-text comments. Participants, identified by anonymous ID numbers, then participated in asynchronous online discussion moderated by the study principal investigator (JM) and RAND co-investigator (DK).

Round 3 began on 17 September 2020. Participants again have the opportunity to review their own and other participants' numerical and free-text comments/discussion from rounds 1 and 2. They are then given an opportunity to change their final numerical responses.

\section{Data analysis}

ExpertLens automatically identifies whether there is consensus, or disagreement, among responses in panels based on decision rules derived a priori using the RAM. ${ }^{212526}$ Specifically, this method uses a two-step analytic approach: first, it identifies disagreement by evaluating the distribution of ratings. If no disagreement exists, it uses the median value to determine whether the panel rating was positive, negative or uncertain (figure 2) ${ }^{21} \mathrm{We}$ used this automatic process to analyse data from rounds 1 and 3 . As round 2 is purely discussion-based, there was no analysis plan. Of note, only round 3 responses will be used to assess final consensus and disagreement. Qualitatively,
Step 1: Calculate Interpercentile Range (IPR)

$$
\text { IPR }=\text { 70th percentile }- \text { 30th percentile }
$$

Step 2: Calculate Interpercentile Range Adjusted for Symmetry (IPRAS)

IPRAS $=2.35+\left(A I^{\star} 1.5\right)$

$\mathrm{Al}=\mathrm{Asymmetry}$ Index: distance between the central point of IPR and central point of rating scale (1-9)

\section{Step 3: Determine the Existence of Disagreement}

- If IPR > IPRAS, there is disagreement

- If IPR < IPRAS, there is no disagreement

- Median score 6.5 to 9: appropriate

- Median score 3.5 to 6: uncertain appropriateness

- Median score 1 to 3: not appropriate

Figure 2 Statistical approach to analysing data about appropriateness of management strategies from a modified Delphi panel..$^{25} 30$

we analysed free-text comments from all rounds using thematic analysis. ${ }^{27}{ }^{28}$ We grouped comments for each strategy by numeric ratings to which they referred. Data were coded by three individuals trained in qualitative analysis and supervised by an expert in these methods (DK) who reviewed all results. Collectively, the team met to discuss coding disagreements until consensus was reached. The lead author (JM) reviewed final codes to ensure correct interpretation of qualitative data clinically.

\section{Patient and public involvement}

No patients involved.

\section{ETHICS AND DISSEMINATION}

This project received ethical approval from the University of Pittsburgh's Institutional Review Board (study 19110301) and the RAND Institutional Research Board (study 2020-0142).

At the conclusion of round 3, our findings can immediately provide guidance to clinicians, especially palliative care clinicians, who provide care for patients with serious illness such as advanced cancer. We will then disseminate this guidance through national networks of stakeholders, and use these as the basis to develop an implementation toolkit that can be used by palliative care clinicians. We will also share results through peer-reviewed publications and at conferences. Results from this modified Delphi study will help inform policy. These could include policies that would reduce barriers to consensus strategies (eg, use of medications for opioid use disorder like increasing availability of buprenorphine/naloxone in settings where 
patients with serious illness are managed) at the clinic, health system, state or federal level.

\section{Author affiliations}

${ }^{1}$ Department of Medicine, Oregon Health \& Science University, Portland, Oregon, USA

${ }^{2}$ Division of General Internal Medicine, Section of Palliative Care and Medical Ethics, University of Pittsburgh School of Medicine, Pittsburgh, Pennsylvania, USA

${ }^{3}$ Survey Research Group, RAND Corporation, Santa Monica, California, USA

${ }^{4}$ Section of Geriatrics, Yale University School of Medicine, New Haven, Connecticut, USA

${ }^{5}$ Division of General Internal Medicine, Center for Research on Health Care, University of Pittsburgh, Pittsburgh, Pennsylvania, USA

${ }^{6}$ Brookdale Department of Geriatrics and Palliative Medicine, Icahn School of Medicine at Mount Sinai, New York, New York, USA

${ }^{7}$ Division of Hematology-Oncology, Feinberg School of Medicine, Northwestern University, Chicago, Illinois, USA

${ }^{8}$ Division of Palliative Care and Geriatric Medicine, Massachusetts General Hospital and Harvard Medical School, Boston, Massachusetts, USA

Contributors JM, DM, RA and JL: fist conceptualised the study idea. JM, RA, JK, JL, DM, JP, CR and DK: developed and piloted cases for subsequent rounds. KC: coordinated the study. ED: managed the data collection activities. CK: wrote the first draft of the protocol. DK: led the analysis and oversaw the use of ExpertLens JM: oversaw the project. All authors will participate in interpretation of findings. All authors reviewed and approved this study protocol.

Funding This study was supported by Cambia Health Foundation (grant number $\mathrm{N} / \mathrm{A})$.

Competing interests This grant was supported by Cambia Health Foundation (PI Dr Merlin).

Patient and public involvement Patients and/or the public were not involved in the design, or conduct, or reporting, or dissemination plans of this research.

Patient consent for publication Not required.

Provenance and peer review Not commissioned; externally peer reviewed.

Open access This is an open access article distributed in accordance with the Creative Commons Attribution Non Commercial (CC BY-NC 4.0) license, which permits others to distribute, remix, adapt, build upon this work non-commercially, and license their derivative works on different terms, provided the original work is properly cited, appropriate credit is given, any changes made indicated, and the use is non-commercial. See: http://creativecommons.org/licenses/by-nc/4.0/.

\section{ORCID iD}

Caroline King http://orcid.org/0000-0002-9913-6340

\section{REFERENCES}

1 Kelley AS, Bollens-Lund E. Identifying the population with serious illness: the "Denominator" challenge. J Palliat Med 2018;21:S-7-16.

2 Rabow MW, Smith AK, Braun JL, et al. Outpatient palliative care practices. Arch Intern Med 2010;170:654-5.

3 Center to Advance Palliative Care. About palliative care, 2020. Available: https://www.capc.org/about/palliative-care/ [Accessed 27 Jan 2021].

4 Swetz KM, Kamal AH. Palliative care. Ann Intern Med 2018;168:ITC33-48.

5 Dowell D, Haegerich TM, Chou R. CDC guideline for prescribing opioids for chronic pain - United States, 2016. MMWR Recomm Rep 2016;65:1-49.

6 Vowles KEet al. Rates of opioid misuse, abuse, and addiction in chronic pain: a systematic review and data synthesis. Pain 2015;156:569-76.
7 Meghani SH, Vapiwala N. Bridging the critical divide in pain management guidelines from the CDC, NCCN, and ASCO for cancer survivors. JAMA Oncol 2018;4:1323-4.

8 Merlin JS, Childers J, Arnold RM. Chronic pain in the outpatient palliative care clinic. Am J Hosp Palliat Care 2013;30:197-203.

9 Taveros MC, Chuang EJ. Pain management strategies for patients on methadone maintenance therapy: a systematic review of the literature. BMJ Support Palliat Care 2017;7:383-9.

10 Vowles KE, McEntee ML, Julnes PS, et al. Rates of opioid misuse, abuse, and addiction in chronic pain: a systematic review and data synthesis. Pain 2015;156:569-76.

11 Merlin JS, Patel K, Thompson N, et al. Managing chronic pain in cancer survivors prescribed long-term opioid therapy: a national survey of ambulatory palliative care providers. J Pain Symptom Manage 2019;57:20-7.

12 National Institutes of Health. Pathways to prevention workshop: the role of opioids in the treatment of chronic pain, 2014.

13 Fitch Ket al. The RAND/UCLA appropriateness method user's manual. RAND Corporation, 2001.

14 Merlin JS, Young SR, Azari S, et al. Management of problematic behaviours among individuals on long-term opioid therapy: protocol for a Delphi study. BMJ Open 2016;6:e011619.

15 Khodyakov D, Grant S, Barber CEH, et al. Acceptability of an online modified Delphi panel approach for developing health services performance measures: results from 3 panels on arthritis research. $J$ Eval Clin Pract 2017;23:354-60.

16 Merlin JS, Young SR, Arnold R, et al. Managing opioids, including misuse and addiction, in patients with serious illness in ambulatory palliative care: a qualitative study. Am J Hosp Palliat Care 2020;37:507-13.

17 Young SR, Azari S, Becker WC, et al. Common and challenging behaviors among individuals on long-term opioid therapy. J Addict Med 2020;14:305-10.

18 Paice JA, Portenoy R, Lacchetti C, et al. Management of chronic pain in survivors of adult cancers: American Society of clinical oncology clinical practice guideline. J Clin Oncol 2016;34:3325-45.

19 National Comprehensive Cancer Network. Adult cancer pain. Available: https://www.nccn.org/professionals/physician_gls/pdf/ pain.pdf [Accessed 28 Sep 2020].

20 Merlin JS, Young SR, Starrels JL, et al. Managing concerning behaviors in patients prescribed opioids for chronic pain: a Delphi study. J Gen Intern Med 2018;33:166-76.

21 Fitch K, Bernstein SJ, Aguilar MD. The RAND/UCLA appropriateness method user's manual. Santa Monica, CA: RAND Corporation, 2001.

22 Ryan K, Gannon-Slater N, Culbertson MJ. Improving survey methods with cognitive interviews in small- and Medium-Scale evaluations. Am J Eval 2012;33:414-30.

23 Khodyakov D, Hempel S, Rubenstein L, et al. Conducting online expert panels: a feasibility and experimental replicability study. BMC Med Res Methodol 2011;11:174.

24 Dalal S, Khodyakov D, Srinivasan R, et al. ExpertLens: a system for eliciting opinions from a large pool of non-collocated experts with diverse knowledge. Technol Forecast Soc Change 2011;78:1426-44.

25 Khodyakov D, Grant S, Denger B, et al. Using an online, modified Delphi approach to engage patients and caregivers in determining the patient-centeredness of duchenne muscular dystrophy care considerations. Med Decis Making 2019;39:1019-31.

26 Khodyakov D, Mikesell L, Schraiber R, et al. On using ethical principles of community-engaged research in translational science. Transl Res 2016;171:52-62.

27 Bengtsson $\mathrm{M}$. How to plan and perform a qualitative study using content analysis. NursingPlus Open 2016;2:8-14.

28 Braun V, Clarke V. Using thematic analysis in psychology. Qual Res Psychol 2006;3:77-101.

29 Corporation, R. How expert lens works. Available: https://www.rand. org/pubs/tools/expertlens/about/how-it-works.html [Accessed 14 Sep 2020].

30 Campbell M, Moore G, Evans RE, et al. ADAPT study: adaptation of evidence-informed complex population health interventions for implementation and/or re-evaluation in new contexts: protocol for a Delphi consensus exercise to develop guidance. BMJ Open 2020;10:e038965. 